\title{
A case of extremely early cervical adenocarcinoma diagnosed only by endocervical curettage with macroscopic pelvic lymph node metastases
}

\author{
Jae Hak Jung, Byoung Ryun Kim \\ Department of Obstetrics and Gynecology, Wonkwang University Hospital, Wonkwang University School of Medicine, Iksan, Korea
}

Microinvasive adenocarcinoma is not as well studied as microinvasive squamous cell carcinoma because diagnosis of adenocarcinoma cannot be ascertained for early invasive lesions. However, most clinicians consider a depth of invasion of $3 \mathrm{~mm}$ without lympho-vascular space invasion as the maximum limit for conservative management. Microinvasive cervical adenocarcinoma is characterized by a rare incidence of lymph node metastasis and very good prognosis. We describe a 62-year-old patient with an extremely early cervical adenocarcinoma which was detected only by endocervical curettage. However, she had multiple macroscopic pelvic node metastases. Clinicians should consider the probability of lymph node metastasis, although management of stage IA1 cervical adenocarcinoma may still be conservative.

Keywords: Cervical cancer; Microinvasive adenocarcinoma; Lymph node metastasis

\section{Introduction}

The prevalence of cervical adenocarcinoma has gradually increased in recent decades; currently, adenocarcinomas comprise $20-25 \%$ of all invasive cervical cancers and $12 \%$ of all microinvasive cervical cancers [1]. Several definitions of microinvasive adenocarcinoma in the cervix have been used. Microinvasive adenocarcinoma is the International Federation of Gynecology and Obstetrics stage 1A, invasive cancer limited to the cervix and identified microscopically. Microinvasive carcinoma of the cervix is subdivided into stages IA 1 , stromal invasion with a measured depth of less than $3 \mathrm{~mm}$ and a width less than $7 \mathrm{~mm}$, and IA2, stromal invasion with a measured depth greater than $3 \mathrm{~mm}$ and less than $5 \mathrm{~mm}$, and a width less than $7 \mathrm{~mm}$ [2].

Many studies have shown that metastasis and recurrence rates are very low in microinvasive cervical adenocarcinoma and the prognosis is generally very good $[3,4]$. Therefore, conservative, non-radical surgery is appropriate for some patients. Microinvasive cancer in women who want to preserve their fertility can be treated by cold knife cone biopsy (conization) and trachelectomy as alternative procedures. Several studies show that these less invasive procedures have no significant differences in survival and recurrence rates, as compared with radical surgery $[3,4]$. Up to now, very few cases of microscopic lymph node metastasis in stage $1 \mathrm{~A} 1$ cervical adenocarcinoma have been reported $[5,6]$. Here, we report for the first time that a case of extremely early cervical adenocarcinoma was detected by preoperative endocervical curettage (ECC) but not diagnosed by postoperative biopsy of entire uterine cervix after hysterectomy with macroscopic pelvic lymph node metastases.

Received: 2019.04.24. Revised: 2019.06.24. Accepted: 2019.06.25. Corresponding author: Byoung Ryun Kim

Department of Obstetrics and Gynecology, Wonkwang University Hospital, Wonkwang University School of Medicine, 895 Muwangro, Iksan 54538, Korea

E-mail: brkim74@wku.ac.kr

https://orcid.org/0000-0002-4213-263X

Articles published in Obstet Gynecol Sci are open-access, distributed under the terms of the Creative Commons Attribution Non-Commercial License (http://creativecommons. org/licenses/by-nc/3.0/) which permits unrestricted non-commercial use, distribution, and reproduction in any medium, provided the original work is properly cited.

Copyright $\odot 2019$ Korean Society of Obstetrics and Gynecology 


\title{
Obstetrics \& Gynecology Science
}

\author{
Vol. 62, No. 6, 2019
}

\section{Case report}

A 62-year-old woman, gravida 3, para 3, abortus 1, was referred to our hospital for further evaluation of an abnormal Pap smear with an adenocarcinoma diagnosis. She had no diseases and no prior adenocarcinoma family history. On bimanual examination, the uterus and both adnexa were normal and parametrial tissues were soft. The patient underwent human papillomavirus (HPV) genotyping and a colposcopy. HPV genotyping was positive for HPV 16, a highrisk HPV type, and the colposcopy finding was unsatisfactory (Fig. 1A). ECC in this patient was performed to evaluate the occult cervical cancer. Only scanty endocervical tissue and mucus material were obtained. The histological findings of the ECC specimen revealed many scattered fragments of adenocarcinoma, which showed atypical cribriform glands with frequent mitotic figures (Fig. 2A and B). However, stromal invasion in this specimen was not identified. The levels of various tumor markers in blood were measured and the following values: squamous cell carcinoma (SCC) antigen $1.0 \mathrm{ng} / \mathrm{mL}$, carbohydrate antigen $12594.6 \mathrm{U} / \mathrm{mL}$.

In the radio-imaging study for cancer staging, the pelvic magnetic resonance imaging (MRI) revealed multiple enlarged lymph nodes with enhancement at the right external and internal iliac nodal stations (Fig. 1B and C), and positron emission tomography-computed tomography (PET-CT) showed intense hypermetabolism on the right external and internal iliac lymph nodes. However, hypermetabolism was not observed in the uterine cervix (Fig. 1D and E). Laparoscopic radical hysterectomy with bilateral salpingo-oopho-
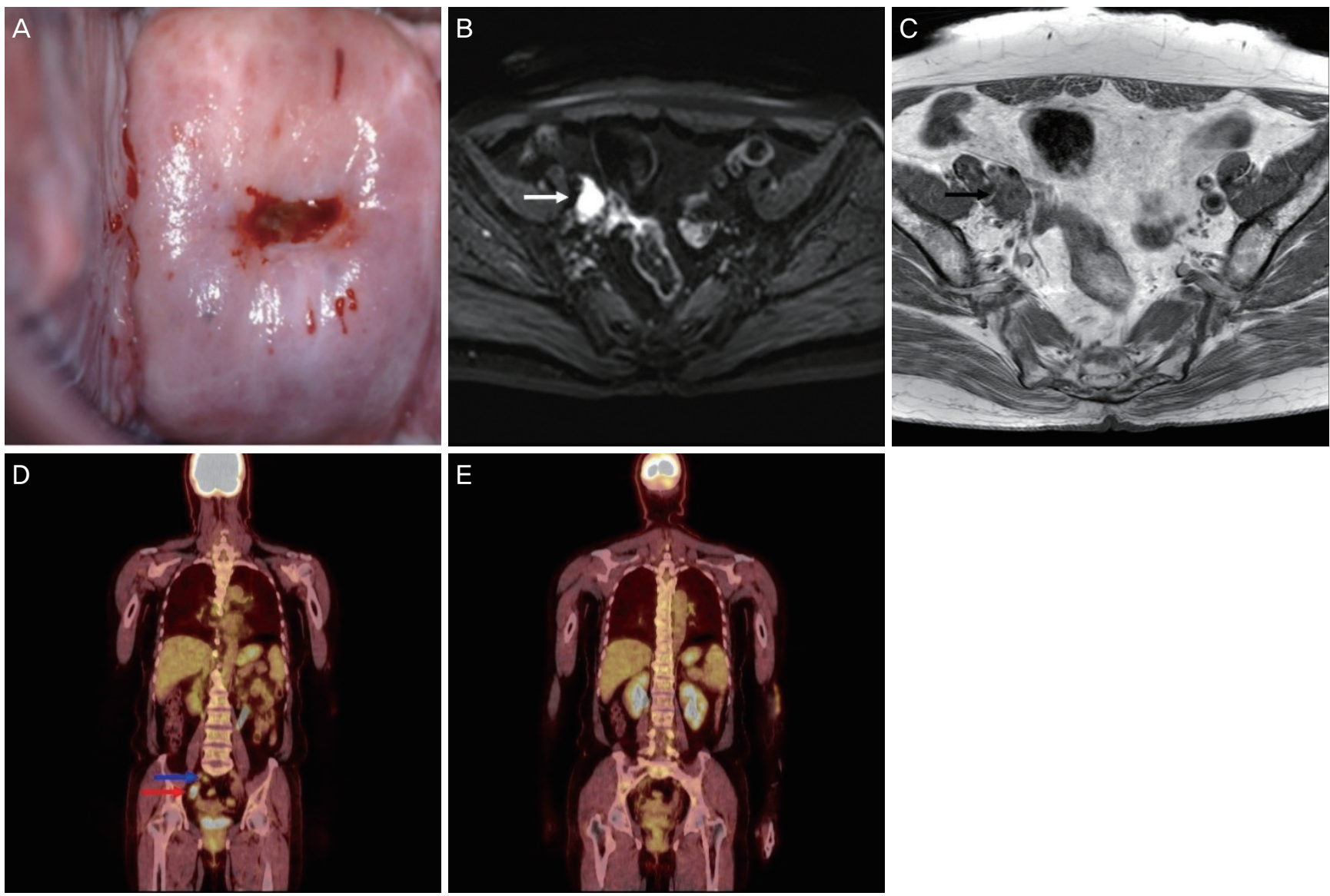

Fig. 1. (A) Colposcopy shows unsatisfactory findings. (B) Pelvic magnetic resonance imaging (MRI) reveals multiple enlarged lymph nodes in the right external iliac nodal station, which show restricted diffusion in a diffusion-weighted image (white arrow). (C) Pelvic MRI shows heterogeneous enhancement on a T1-weighted contrast enhanced image (black arrow). (D) Positron emission tomography-computed tomography (PET-CT) reveals intense hypermetabolism on the right external iliac nodal station (red arrow) and internal iliac nodal station (blue arrow). (E) PET-CT reveals no hypermetabolic lesion on the uterine endocervix. 


\section{Obstetrics \& Gynecology Science}

Jae Hak Jung, et al. Early adenocarcinoma of uterine cervix

rectomy (BSO) and pelvic and para-aortic lymphadenectomy was performed after discussing treatment options with our patient.

External and internal iliac lymph nodes of approximately $2 \mathrm{~cm}$ were observed intraoperatively. A frozen section of these pelvic lymph nodes was suspicious for metastatic adenocarcinoma. The surgery was finished without any complications and the patient had an uneventful postoperative course. A histological examination revealed multiple metastatic pelvic lymph nodes (right external, internal, and common iliac and obturator lymph nodes) (Fig. 2C). The exocervix and endocervix were radially sectioned and entirely submitted for histologic assessment, which revealed no cancer tissue in the uterine cervix and uterine corpus.

She was subsequently treated with adjuvant concurrent chemoradiation therapy with cisplatin $40 \mathrm{mg} / \mathrm{m}^{2}$ intravenously once per week for 6 weeks and whole pelvic radiation. After 24 months of follow-up, she continued to be diseasefree.

\section{Discussion}

Cervical adenocarcinoma incidence has been increasing in recent decades [1]. Despite this increase, there are few reports of cervical adenocarcinoma. Most of the characteristics of cervical adenocarcinoma are similar to SCC. However, in comparison to early-stage SCC, cervical adenocarcinoma has a higher mortality rate and a higher risk of metastasis when tumor size is larger than $4 \mathrm{~cm}$; also, HPV 18-positive tumors are more likely to be cervical adenocarcinoma [7-9]. Most studies have reported early-stage cervical adenocarcinoma but there are few reports on microinvasive cervical adenocarcinoma.

In the diagnosis of abnormal cytology findings, a directed biopsy through colposcopy has been commonly used. When endocervical lesions are suspected, many colposcopists perform ECC to increase the reliability and reduce the risk of occult cancer [10]. ECC is a procedure for scraping the non-visualized area of the endocervical canal using a spoon-shaped device called a curette. Invasive cervical adenocarcinoma is very problematic for diagnosing superficially invasive lesions as well as limited (superficial) biopsy specimens (e.g., ECC). As opposed to squamous carcinoma, invasion of adenocarcinoma may not be accompanied with a significant stroma response. Verification of invasion of adenocarcinoma is based on the presence of a significant infiltrative glandular pattern, serious glandular irregularity, and complex neoplastic glandular form adjacent to the normal endocervical crypts [11].

The extremely early adenocarcinoma in this case was diagnosed only by ECC and the treatment plan was determined after staging workup. Due to optional testing modalities revealing multiple pelvic lymph node metastases, we performed laparoscopic radical hysterectomy with BSO and pelvic and para-aortic lymphadenectomy. Although the cervix was entirely submitted for histologic evaluation after surgery, cancer tissue was not detected. However, multiple metastatic pelvic lymph nodes (right external, internal, and common iliac and obturator lymph nodes) were confirmed.

Microinvasive cervical adenocarcinoma has a significantly low recurrence rate and lymph node metastasis rate. According to a meta-analysis by Reynolds et al. [5], out of 37 cases with IA1 and 4 cases with IA2 that underwent conization, none had lymph node metastases or recurrence. Additionally,
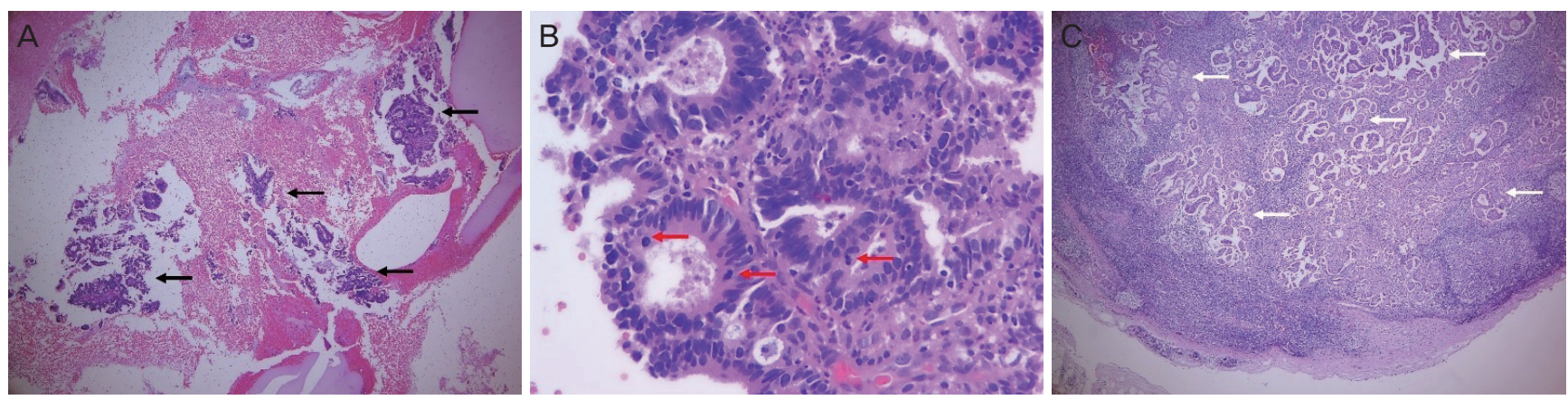

Fig. 2. (A) A photomicrograph shows many scattered fragments of adenocarcinoma in the curettage specimen (black arrow) (hematoxylin and eosin $[H \& E], 40 \times)$. (B) A photomicrograph shows atypical cribriform glands with frequent mitotic figures (red arrow) (H\&E, 400×). (C) A photomicrograph shows many clusters of metastatic adenocarcinoma in the pelvic lymph nodes (white arrow) (H\&E, 40×). 


\section{Obstetrics \& Gynecology Science}

Vol. 62, No. 6, 2019

among the 210 patients who underwent lymphadenectomy, only 2 patients had lymph node metastases and these were microscopic. Based on this meta-analysis, conservative management of cervical adenocarcinoma by using trachelectomy or conization can be performed to preserve fertility.

Nagarsheth et al. [6] reported a rare case of microinvasive cervical adenocarcinoma with microscopic lymph node metastasis. The patient underwent a curettage and the histologic findings were diagnosed as malignant. She was treated with a total abdominal hysterectomy with BSO and bilateral pelvic lymphadenectomy. Histologic examination of the cervix showed an invasive endometrioid adenocarcinoma of the cervix. The depth of invasion was $2.5 \mathrm{~mm}$, the largest horizontal dimension was $4.0 \mathrm{~mm}$ and microscopic pelvic lymph nodal metastases were identified.

These previous cases histologically identified stromal invasion but no stromal invasion was identified in our patient. We report a new case of extremely early cervical adenocarcinoma with multiple macroscopic pelvic lymph node metastases, highlighting the probability of nodal metastases in the case of early invasive adenocarcinoma. Stage IA1 adenocarcinomas may be managed conservatively; however, clinicians must consider optional testing modalities (e.g., CT, MRI, and PET-CT) in order to identify a possible lymph node metastasis.

\section{Acknowledgments}

This work was supported by a research grant from Wonkwang University received in 2018.

\section{Conflict of interest}

No potential conflict of interest relevant to this article was reported.

\section{Patient consent}

The patient provided written informed consent for the publication and the use of her images.

\section{References}

1. Sherman ME, Wang SS, Carreon J, Devesa SS. Mortality trends for cervical squamous and adenocarcinoma in the United States. Relation to incidence and survival. Cancer 2005;103:1258-64.

2. Pecorelli S. Revised FIGO staging for carcinoma of the vulva, cervix, and endometrium. Int I Gynaecol Obstet 2009;105:103-4.

3. Baalbergen A, Smedts F, Helmerhorst TJ. Conservative therapy in microinvasive adenocarcinoma of the uterine cervix is justified: an analysis of 59 cases and a review of the literature. Int I Gynecol Cancer 2011;21:1640-5.

4. Bean LM, Ward KK, Plaxe SC, McHale MT. Survival of women with microinvasive adenocarcinoma of the cervix is not improved by radical surgery. Am J Obstet Gynecol 2017;217:332.e1-332.e6.

5. Reynolds EA, Tierney K, Keeney GL, Felix JC, Weaver AL, Roman LD, et al. Analysis of outcomes of microinvasive adenocarcinoma of the uterine cervix by treatment type. Obstet Gynecol 2010;116:1150-7.

6. Nagarsheth NP, Maxwell GL, Bentley RC, Rodriguez G. Bilateral pelvic lymph node metastases in a case of FIGO stage IA(1) adenocarcinoma of the cervix. Gynecol Oncol 2000;77:467-70.

7. Galic V, Herzog TJ, Lewin SN, Neugut Al, Burke WM, Lu YS, et al. Prognostic significance of adenocarcinoma histology in women with cervical cancer. Gynecol Oncol 2012;125:287-91.

8. Lee YY, Choi CH, Kim TJ, Lee JW, Kim BG, Lee JH, et al. A comparison of pure adenocarcinoma and squamous cell carcinoma of the cervix after radical hysterectomy in stage IB-IIA. Gynecol Oncol 2011;120:439-43.

9. Im SS, Wilczynski SP, Burger RA, Monk BJ. Early stage cervical cancers containing human papillomavirus type 18 DNA have more nodal metastasis and deeper stromal invasion. Clin Cancer Res 2003;9:4145-50.

10. Akladios C, Lecointre L, Baulon E, Thoma V, Averous $\mathrm{G}$, Fender $\mathrm{M}$, et al. Reliability of endocervical curettage in the diagnosis of high-grade cervical neoplasia and cervical cancer in selected patients. Anticancer Res 2015;35:4183-9.

11. Holschneider CH, Berek JS. Valvar cancer. In: Berek JS, Novak E, editors. Berek \& Novak's gynecology. 15th ed. Philadelphia (PA): Lippincott Williams \& Wilkins; 2014. p.135-7. 